\title{
IL-1, IL-6, TNF-a, and MIF in relative adrenal insufficiency in septic shock: a piglet model
}

Bina Akura, ${ }^{1}$ Jose R. L. Batubara, ${ }^{2}$ Zakiudin Munasir, ${ }^{2}$ Gunanti, ${ }^{3}$ Joedo Prihartono, ${ }^{4}$ Hindra Irawan Satari, ${ }^{2}$ Suzanna Immanuel, ${ }^{5}$ Dadang Hudaya Somasetia ${ }^{6}$

Check for updates

pISSN: 0853-1773 • elSSN: 2252-8083 https://doi.org/10.13181/mji.oa.202943 Med J Indones. 2020;29:250-9

Received: February 08, 2019

Accepted: June 08, 2020

Authors' affiliations:

${ }^{1}$ Fatmawati Hospital, Jakarta, Indonesia, ${ }^{2}$ Department of Pediatric, Faculty of Medicine, Universitas Indonesia, Jakarta, Indonesia, ${ }^{3}$ Department of Surgery and Radiology, Faculty of Veterinary Medicine, Institut Pertanian Bogor, Bogor, Indonesia, ${ }^{4}$ Department of Community Medicine, Faculty of Medicine, Universitas Indonesia, Jakarta, Indonesia, ${ }^{5}$ Department of Clinical Pathology, Faculty of Medicine, Universitas Indonesia, Jakarta, Indonesia, ${ }^{6}$ Department of Pediatric, Faculty of Medicine, Universitas Padjadjaran, Bandung, Indonesia

\section{Corresponding author:}

Bina Akura

Fatmawati Hospital, Jalan RS. Fatmawati Raya No.4, Cilandak Barat, Cilandak, South Jakarta 12430, DKI Jakarta,

Indonesia

Tel/Fax: +62-21-7501524/

$+62-21-7690123$

E-mail: binaakura@gmail.com

\begin{abstract}
BACKGROUND Inflammatory mediators released during septic shock are involved in the mechanism of adrenal insufficiency. This study investigated the role of interleukin (IL)-6, IL-1, tumor necrosis factor (TNF)- $\alpha$, and macrophage migration inhibitory factor (MIF) in septic shock with relative adrenal insufficiency (RAI).
\end{abstract}

METHODS We conducted a 6-month experimental study in 20 piglets. Following endotoxin administration, their hemodynamics were monitored and blood samples were drawn to test the levels of cytokines IL-1, IL-6, TNF- $\alpha$, and MIF every 15 min until septic shock onset as well as during a corticotropin stimulation test. Septic shock was managed by administering fluid resuscitation, inotropic drugs, and hydrocortisone. At the end of the study, the piglet models were classified as either RAI or non-RAI. Immunohistochemistry staining was performed on the hypothalamus of the RAI group.

RESULTS The level of IL- 6 at 45 min was higher in the RAI group than the non-RAI group $(p=0.008)$, and that of IL-1 was similar in the two groups during septic shock. The RAI group had higher TNF- $\alpha$ levels at $15 \min (p=0.002)$ and at $30 \mathrm{~min}(p=0.007)$ than the non-RAI group, and the MIF level during septic shock was higher in the RAI group $(p=0.003)$ than the non-RAI group.

CONCLUSIONS Cytokine-induced inflammatory process of adrenal gland reflected in TNF-a level in $15 \mathrm{~min}$ and $30 \mathrm{~min}$, IL-6 in $45 \mathrm{~min}$, and MIF in septic shock condition but not in IL-1.

KEYWORDS adrenal insufficiency, interleukin-1, interleukin-6, macrophage migration inhibition factors, septic shock, tumor necrosis factor-alpha
Sepsis refers to a dysfunction that can be lifethreatening and is caused by immune dysregulation in response to infection. ${ }^{1,2}$ Sepsis diagnosis is based on a predisposition to infection, signs of ongoing infection, inflammation responses, and organ dysfunction or failure. ${ }^{1}$ Management of sepsis in "Surviving Sepsis Campaign" has established an early diagnosis and prompt administration of adequate antibiotic treatment and resuscitation are the only measures that improve the prognosis. ${ }^{2}$ Despite these advances, the mortality of children with septic shock in developed countries is still above $12 \% .^{2}$ Incidence adrenal insufficiency in sepsis patients is $30.7 \%$ in Brazil.3 At Cipto Mangunkusumo hospital in 2009, there were 61 cases of septic shock and 28 cases (45.9\%) were death. ${ }^{4}$ Neuroendocrine dysfunction could occur in patients with severe sepsis or septic shock. ${ }^{5}$ Adrenal insufficiency in septic shock is 
temporary, and conditions return to normal with the improvement of sepsis. Thus, it is suspected that inflammatory mediators released during septic shock cause hypothalamus-pituitary-adrenal (HPA) axis dysfunction and may play a role in the development of adrenal insufficiency. ${ }^{5}$

Cytokines mediate the immune/metabolic response to external stimuli and the transition from sepsis to septic shock and then to multiple organ dysfunction syndrome.5,6 The mechanism of relative adrenal insufficiency (RAI) in septic shock is exceptionally complex. It is suspected that inflammatory mediators released during septic shock play a role in HPA axis dysfunction and in the mechanism of adrenal insufficiency., ${ }^{5,6}$ In addition to their stimulatory function, the release of inflammatory mediators during sepsis could also inhibit the synthesis and release of cortisol via the action of cytokines at the HPA axis and at glucocorticoid receptors. Interleukin (IL)-6 and IL-1 stimulate cortisol secretion by affecting the components of the HPA axis.5,6 Another immune mediator, macrophage migration inhibitory factor (MIF), is secreted by the anterior pituitary gland and macrophages to counteract glucocorticoid action. ${ }^{7}$ Because the incidence of RAI remains high, it is crucial to determine which cytokines among IL-1, IL-6, tumor necrosis factor (TNF)- $\alpha$, and MIF play an important role, and this has yet to be investigated. However, it would be unethical to perform such a study in humans. In such cases, studies have been performed using Sus scrofa, due to similarities with humans in terms of physiology, anatomy, and biochemical structure..$^{8-10}$ The primary objectives of this study were to investigate the role of cytokines IL-1, IL-6, TNF- $a$, and MIF in the development of RAI in septic shock and to observe the histopathological features in the adrenal glands, pituitary glands, and hypothalamus in a piglet model of septic shock with RAI.

\section{METHODS}

\section{Study design}

This 6-month experimental study (AprilSeptember 2015) was conducted in the Experimental Surgery Laboratory, Surgery and Radiology Division, Reproduction Clinic and Department of Pathology, Faculty of Veterinary, Institut Pertanian Bogor (IPB). We also used the facilities at the integrated laboratory of the Faculty of Medicine, Universitas Indonesia, and the
IPB Primate Laboratory. Ethical approval was obtained from the Medical/Health Research Ethics Committee of the Faculty of Medicine, Universitas Indonesia/Cipto Mangunkusumo Hospital (No: 939/UN2.F1/ETIK/X/2015) and the Animal Ethics Committee of the Faculty of Veterinary, IPB (No: 026/KEH/SKE/IV/2015).

\section{Sample selection}

We used 20 piglets (Sus scrofa) from two healthy litters aged 6-8 weeks with a bodyweight of 5-10 $\mathrm{kg}$, equivalent to the weight of infant and pediatric patients aged 3 months-2 years. A sample size of 20 was calculated using a single proportion formula with $a=0.05$, a proportion of 0.3 , and precision of $0.2 .^{10}$

\section{Inclusion and exclusion criteria}

Piglets were only included if they were declared healthy by the veterinarian, with normal standard physical, blood, blood gas analysis, and chest X-ray examinations. Piglets were excluded if the veterinarian detected abnormal heart sounds or lung abnormalities upon X-ray. Those piglets that did not complete the study protocol or died before all study procedures were completed, those with incomplete laboratory results, and those with unstable hemodynamics were excluded.

\section{Pre-study screening}

A physical diagnostic examination was conducted by a veterinarian prior to study commencement, including routine blood examinations, blood gas analysis, and a chest X-ray. The piglets were given the antibiotic enrofloxacin for treatment against Escherichia coli and Salmonella, and oxfendazole for deworming.

\section{Study procedure}

The study procedure included sedation/ anesthesia, intubation, vascular access, sampling, a corticotropin stimulation test (Synacthen ${ }^{\circledR}$ ), and pathological examination of the adrenal glands, pituitary glands, and hypothalamus. Anesthesia was performed using a combination of $20 \mathrm{mg} / \mathrm{kg}$ body weight (BW) 10\% ketamine $\mathrm{HCl}$ and $2 \mathrm{mg} / \mathrm{kgBW} 10 \%$ xylazine $\mathrm{HCl}$ administered intramuscularly in the neck muscles, followed by $1 \%$ propofol (100-300 ug/kgBW/ min) and phenthanyl ( $2 \mathrm{mcg} / \mathrm{kgBW} /$ hour $).{ }^{10} \mathrm{~A}$ central arterial catheter (Pulsiocath PV2015, Pulsion Medical Systems, Germany) was inserted into descending aorta 
through the femoral artery, and a venous catheter (Certofix ${ }^{\circledR}$ Duo Paed, B-Braun, Germany) was inserted deep into the jugular vein. Then, they were attached to a pressure transducer and pulse index continuous cardiac output (PiCCO) device (B850 General Electric, USA $)^{10}$ to measure arterial blood pressure, mean arterial pressure (MAP), and cardiac index $(\mathrm{Cl})$. The heart and respiratory rates were also monitored. The heart rate, respiratory rate, temperature, MAP, and $\mathrm{Cl}$ was determined for all piglets. The white blood cells count and levels of IL-1, IL-6, TNF- $\alpha$, MIF, cortisol, and adrenocorticotropin hormone (ACTH) were measured prior to study procedure, during sepsis, and during septic shock. Blood gas analysis was also performed. The blood glucose level was measured using glucometer (GlucoDr, Allmedicus, Republic of Korea) and the lactate level was measured using i-STAT (Abbott Point of Care Inc., Abbott Park, USA). A PiCCO monitor (B850 General Electric) was used to measure heart rate, MAP, and $\mathrm{Cl}$ to determine the septic shock state.

All animals were given $50 \mu \mathrm{gg} / \mathrm{kgBW}$ lipopolysaccharide (LPS) endotoxin intravenously (E. coli O111:B4; Sigma-Aldrich St. Louis, USA). ${ }^{10}$ The piglets were intensively monitored using a PiCCO device until septic shock occurred. If septic shock had not occurred within 4 hours, a further dose of $1 \mu \mathrm{g} /$ kgBW/hour of E. coli LPS was given. ${ }^{10}$ Blood samples were taken for IL-1, IL-6, TNF- $\alpha$, MIF, ACTH, and cortisol analysis. The samples were drawn before the endotoxin administration and every 15 min thereafter until septic shock occurred. Sepsis signs included increasing or decreasing body temperature, vomiting, and increased heart rate, MAP, white blood cells, and lactate levels. ${ }^{8,11}$ Septic shock was considered to have occurred if the $\mathrm{Cl}$ was $<3.0 \mathrm{I} / \mathrm{min} / \mathrm{m}^{2}{ }^{8,10}$ Septic shock was managed by administering fluid resuscitation, inotropic drugs. Hydrocotisone was given to overcome RAl after giving inotropic drugs and resuscitation.

A corticotropin stimulation test was performed after septic shock had occurred using an injection of synthetic corticotropin. The pre- and post-test cortisol levels were measured using an enzyme-linked immunosorbent assay (ELISA) cortisol kit (Abcam, UK; catalog number: ab108665).

After completing the study procedure, euthanasia was performed with the intravenous administration of $400 \mathrm{mg}$ phenobarbital in the auricular vein, and the adrenal gland, pituitary gland, and hypothalamus were resected for histopathological evaluation. The piglet was grouped into RAI or non-RAI group based on the result of corticotropin stimulation test. All hemodynamic parameters, cytokine levels, and hormone levels were assessed against normal piglet values. ${ }^{8,9}$

\section{Corticotropin stimulation test}

Piglets were grouped based on corticotropin stimulation test at the end of intervention, after we got laboratory result of cortisol. A corticotropin stimulation test was performed by injecting $250 \mathrm{mg}$ (equivalent to $25 \mathrm{IU}$ ) of corticotropin intravenously and followed by flushing with $0.9 \% \mathrm{NaCl}(1 \mathrm{ml} /$ kgBW; Synacthen Depot®; Novartis Pharma $\mathrm{GmbH}$, Germany). ${ }^{9} \quad$ The corticotropin was injected immediately after blood specimen for the pre-test cortisol was drawn $\left(t_{0}\right)$. The post-test sample was taken at 30-60 min after the corticotropin injection. Elevation of the post-test cortisol level ( $\Delta$ max) was calculated as the difference between the cortisol level post corticotropin stimulation test $\left(t_{30-60}\right)$ and pre-test cortisol level $\left(t_{0}\right) \cdot{ }^{9}$ RAI was grossly defined as basal cortisol $<5 \mu \mathrm{g} / \mathrm{dl}$, a cortisol level after synacthen stimulation of $<18 \mu \mathrm{g} / \mathrm{dl}$, or $\Delta$ cortisol $<9 \mu \mathrm{g} / \mathrm{dl} .{ }^{9}$

\section{Laboratory examination}

After collecting blood from the piglets into ethylenediaminetetraacetic acid tubes, they were centrifuged for $15 \mathrm{~min}$ at 1,000 $\times \mathrm{g}$ within $30 \mathrm{~min}$ of collection before being stored at $\leq-20^{\circ} \mathrm{C}$. All laboratory tests were performed at Prodia Laboratory, Jakarta. A Human IL-1 Quantikine ELISA Kit (R\&D Systems, USA) was used to measure the levels of IL-1. ${ }^{12}$ All reagents, working standards, and samples were prepared according to the manufacturer's instructions. Briefly, after adding assay diluent RD-1 to each well of a 96well plate, $200 \mu \mathrm{l}$ of standard, sample, or control was added to each well, covered with an adhesive strip, and incubated for 2 hours at room temperature. Thereafter, each well was aspirated and washed three times with wash buffer $(400 \mu \mathrm{l})$. After the last wash, any remaining wash buffer was removed via aspiration. Then, $200 \mu \mathrm{l}$ of human IL-1 conjugate was added to each well, incubated again for 2 hours, aspirated, and washed as before. Substrate solution $(200 \mu \mathrm{l})$ was added to each well and incubated for 20 min while protected from light. Finally, $50 \mu \mathrm{l}$ of stop solution was added. A microplate reader was used 
to determine the optical density at $450 \mathrm{~nm}$ for each well within $30 \mathrm{~min}$. A Human IL-6 Quantikine ELISA Kit, Human TNF-a Quantikine ELISA Kit, and Human MIF Quantikine ELISA Kit (R\&D Systems) were used to measure IL-6, TNF- $\alpha$, and MIF, respectively, and the procedure used was similar to that described for IL1. ${ }^{12}$ A cortisol ELISA kit and an ACTH immunoassay kit were used to measure the cortisol and ACTH levels, respectively. ${ }^{12}$

\section{Histopathology examination}

The adrenal gland, pituitary gland, and hypothalamus tissue were examined at the IPB Primate Laboratory. The tissues were stained with hematoxylin and eosin for histopathological examination. Immunohistochemical analysis was performed using anti IL-1 antibody [EPR21086] (Abcam 9722), anti IL-6 antibody [EPR21711] (Abcam 229381), anti TNF- $\alpha$ antibody [EPR19147] (Abcam 183218), and anti MIF antibody [EPR 12463] (Abcam 175189).

\section{Statistical analysis}

All data were analyzed using SPSS version 20 (IBM Corp., USA), and the results were presented as text, tables, or graphs. We used a $95 \%$ confidence interval and a power of $80 \%$, and $p$-values $<0.05$ were considered statistically significant. Data with a normal distribution were presented as the mean and standard

Table 1. Variables in baseline and during septic shock between the RAI and non-RAI groups

\begin{tabular}{|c|c|c|c|c|c|}
\hline \multirow[b]{2}{*}{ Variable } & \multicolumn{2}{|c|}{ Baseline } & \multicolumn{2}{|c|}{ During septic shock } & \multirow[b]{2}{*}{$p^{*}$} \\
\hline & $\begin{array}{c}\text { RAI group, } \\
\text { mean (SD) }(N=12)\end{array}$ & $\begin{array}{l}\text { Non-RAI group, } \\
\text { mean }(S D)(N=7)\end{array}$ & $\begin{array}{c}\text { RAI group, } \\
\text { mean (SD) }(N=12)\end{array}$ & $\begin{array}{l}\text { Non-RAI group, } \\
\text { mean }(S D)(N=7)\end{array}$ & \\
\hline Male sex, (n) & 6 & 3 & - & - & - \\
\hline Weight (kg) & $7.8(1.38)$ & $7.6(1.37)$ & - & - & - \\
\hline Length $(\mathrm{cm})$ & $70.3(5.02)$ & $70.7(4.98)$ & - & - & - \\
\hline Heart rate (bpm) & $91.6(13.30)$ & $81.0(10.26)$ & $118.6(21.17)$ & $116.4(24.89)$ & 0.800 \\
\hline \multicolumn{6}{|l|}{ Blood pressure (mmHg) } \\
\hline Systolic & 72.9 (14.18) & 74.1 (9.49) & $54.2(12.92)$ & $54.3(19.14)$ & 0.384 \\
\hline Diastolic & 40 (11.78) & 40 (11.12) & $35.6(10.1)$ & $32.3(8.05)$ & 0.196 \\
\hline $\mathrm{MAP}(\mathrm{mmHg})$ & $51.0(11.47)$ & $51.4(10.41)$ & $43(10.16)$ & $40(4.80)$ & 0.482 \\
\hline Respiratory rate (per min) & $31.2(2.86)$ & $29.6(5.59)$ & $38.8(4.73)$ & $38.2(9.32)$ & 0.219 \\
\hline Temperature $\left({ }^{\circ} \mathrm{C}\right)$ & $33.2(1.77)$ & $33.1(1.41)$ & $36.0(1.74)$ & $34.3(1.73)$ & 0.056 \\
\hline Hemoglobin (g/dl) & $11.7(1.43)$ & $11.0(0.74)$ & $11.6(1.52)$ & $11.1(0.97)$ & 0.711 \\
\hline Hematocrit (\%) & $34.7(5.12)$ & $34.7(3.04)$ & $34.8(3.98)$ & $33.4(3.31)$ & 0.536 \\
\hline WBCs $(\mu l)$ & $12,475(5,483.7)$ & $11,386(5,599.7)$ & $13,925(471.1)$ & $12,157(5,590.7)$ & 0.494 \\
\hline Platelet $\left(10^{3} / \mu \mathrm{l}\right)$ & $427.4(90.02)$ & $364.4(81.93)$ & $351.6(94.11)$ & $353.9(56.42)$ & 0.902 \\
\hline Lactate level (mg/dl) & $1.08(0.27)$ & $1.26(0.50)$ & $1.43(0.44)$ & $2.40(1.82)$ & 0.967 \\
\hline Cortisol level ( $\mu \mathrm{g} / \mathrm{dl})$ & $11.25(6.91)$ & $17.78(6.72)$ & $11.25(6.91)$ & $14.04(7.16)$ & 0.703 \\
\hline $\begin{array}{l}\text { IL-1 (pg/dl), } \\
\text { median (min-max) }\end{array}$ & $15.66(9.99-55)$ & $20.07(7.77-56.54)$ & $19.45(7.77-51.47)$ & $16.28(9.67-67.12)$ & 0.612 \\
\hline $\begin{array}{l}\text { IL-6 (pg/dI), } \\
\text { median (min-max) }\end{array}$ & $0.62(0.51-1.35)$ & $0.56(0.51-0.62)$ & $0.635(0.55-1.23)$ & $0.64(0.57-0.98)$ & 0.701 \\
\hline $\begin{array}{l}\text { TNF- } \alpha \text { (pg/dl), } \\
\text { median (min-max) }\end{array}$ & $34.77(12.32-68.75)$ & 41.39 (16.54-165) & $\begin{array}{c}4,731.03(3,754.52- \\
5,981.97)\end{array}$ & $\begin{array}{c}5,697.72(3,963.06- \\
5,986.97)\end{array}$ & 0.175 \\
\hline $\begin{array}{l}\text { MIF (ng/dl), } \\
\text { median (min-max) }\end{array}$ & $10(8.12-17.3)$ & $15.10(8.17-21.52)$ & $25.28(18.45-30.64)$ & $11.30(7.1-15.14)$ & 0.003 \\
\hline ACTH level (pg/dl) & $10.82(1.30)$ & $11.21(0.64)$ & $10.31(0.42)$ & $10.53(0.46)$ & 0.396 \\
\hline
\end{tabular}

$\mathrm{RAI}=$ relative adrenal insufficiency; $\mathrm{SD}=$ standard deviation; bpm=beats per minute; MAP=mean arterial pressure; WBCs=white blood cells; IL=interleukin; TNF- $\alpha=$ tumor necrosis factor- $\alpha$; MIF=macrophage migration inhibitory factor; ACTH=adrenocorticotropin hormone ${ }^{*} p<0.05$ were considered statistically significant 
Table 2. Corticotropin stimulation test during septic shock

\begin{tabular}{lccc}
\hline Variable & RAl group, mean $(\mathrm{SD})(\mathrm{N}=12)$ & Non-RAl group, mean $(\mathrm{SD})(\mathrm{N}=7)$ & $p^{*}$ \\
\hline Basal cortisol level $(\mu \mathrm{g} / \mathrm{dl})$ & $11.25(6.9)$ & $14.04(7.18)$ & 0.703 \\
Cortisol level $30 \mathrm{~min}(\mu \mathrm{g} / \mathrm{dl})$ & $17.78(6.72)$ & $28.25(8.99)$ & $\mathbf{0 . 0 1 4}$ \\
Cortisol level $60 \mathrm{~min}(\mu \mathrm{g} / \mathrm{dl})$ & $18.18(6.93)$ & $58.03(48.97)$ & $\mathbf{0 . 0 0 9}$ \\
\hline
\end{tabular}

$\mathrm{RAI}=$ relative adrenal insufficiency; $\mathrm{SD}=$ standard deviation

* $p<0.05$ were considered statistically significant

deviation, while data with abnormal distribution were presented as median values. The Mann-Whitney $U$ test, Pearson's correlation coefficient, and Spearman's rank correlation coefficient were used.

\section{RESULTS}

\section{Characteristics of the RAI and non-RAI groups}

All piglets showed septic shock $60 \mathrm{~min}$ after endotoxin administration, except for one, where septic shock only occurred at $105 \mathrm{~min}$, and it was consequently excluded from the analysis. Thus, only 19 subjects ( 9 males and 10 females) were analyzed. The initial cortisol level was $11.25(6.91) \mu \mathrm{g} / \mathrm{dl}$ in the RAI group and 17.78 (6.72) $\mu \mathrm{g} / \mathrm{dl}$ in the non-RAl group. The characteristics of each group are shown in Table 1.

\section{Characteristics of the RAI and non-RAI groups during septic shock}

The characteristics of the RAI and non-RAI groups during the septic shock condition are presented in Table 1. An increased heart rate, respiratory rate, and body temperature were observed in both groups. The mean white blood cell count was slightly increased in both the RAI and non-RAI groups, and the mean levels of IL-1, IL-6, and ACTH were similar in both groups. The cortisol level in the RAI group $11.25(6.91) \mu g / d l$ was lower than in the non-RAl group $14.04(7.16) \mu g / d l$ during septic shock. However, after the corticotropin stimulation test, the RAl group had a lower cortisol level than the non-RAl group (Table 2). Based on the corticotropin stimulation test result, 12/19 (63\%) piglets were considered to have RAI.

\section{Comparison of IL-1, IL-6, TNF- $a$, MIF, cortisol, and ACTH levels between the RAI and non-RAI groups}

Figures 1 shows the dynamic changes of IL-1, IL-6, TNF- $\alpha$, MIF, cortisol, and ACTH in the RAI and non-RAI group. The level of IL- 6 in the RAI group at $45 \mathrm{~min}$ was higher than that of non-RAl group (0.65 [0.5-
4.32] versus $0.54[0.51-0.61] \mathrm{pg} / \mathrm{dl} ; p=0.008)$. The level of IL-6 after synacthen administration was not significantly different between both groups. The level of IL-1 at baseline and at 15-60 min during septic shock was not significantly different between both groups. At 30 and 60 min after synacthen administration, the level of IL-1 was similar in both groups (16.03 [11.56590.82] versus 14.21 [10.57-31.24] and 20.35 [11.97741.37] versus 15.83 [8.3-68.63] pg/dl). The TNF- $\alpha$ level at 15 min during septic shock in the RAI group had a $p$-value of 0.002 when compared with the nonRAl group. The level of TNF- $a$ at 30 min had a $p$-value of 0.007 when compared with the non-RAI group. The MIF level during septic shock in the RAI group compared with the non-RAl group showed a $p$-value of 0.003. After the corticotropin stimulation test, the level of MIF at 30 and 60 min in the RAI group was also similar compared with the non-RAI group (12.46 [8.03-23.32] versus 15.96 [7.7-19.88] and 11.12 [8.07$20.79]$ versus $15.41[7.29-22.12] \mathrm{ng} / \mathrm{dl})$.

\section{Correlation of IL-1, IL-6, TNF- $\alpha$, and MIF with ACTH and cortisol}

IL-6 levels were positively correlated with ACTH levels at $60 \mathrm{~min}(p=0.002, r=0.662)$ but did not correlate with ACTH prior to sepsis induction until 45 min and also after the corticotropin stimulation test. IL-6 was positively correlated with the cortisol level at $15 \min (p=0.008, r=0.586)$ and $60 \min (p=0.008$, $r=0.590)$.

IL-1 levels did not significantly increase the ACTH and cortisol levels during septic shock ( $p=0.994$ and $p=0.258$, respectively) and at $60 \mathrm{~min}$ after the synacthen test ( $p=0.078$ and $p=0.912$, respectively) but were positively correlated the cortisol level during the sepsis condition $(p<0.001, r=0.728)$. TNF- $\alpha$ did not significantly increase the ACTH and cortisol levels during septic shock ( $p=0.887$ and $p=0.545$, respectively) and at $60 \mathrm{~min}$ after the synacthen test ( $p=0.980$ and $p=0.124$, respectively). 

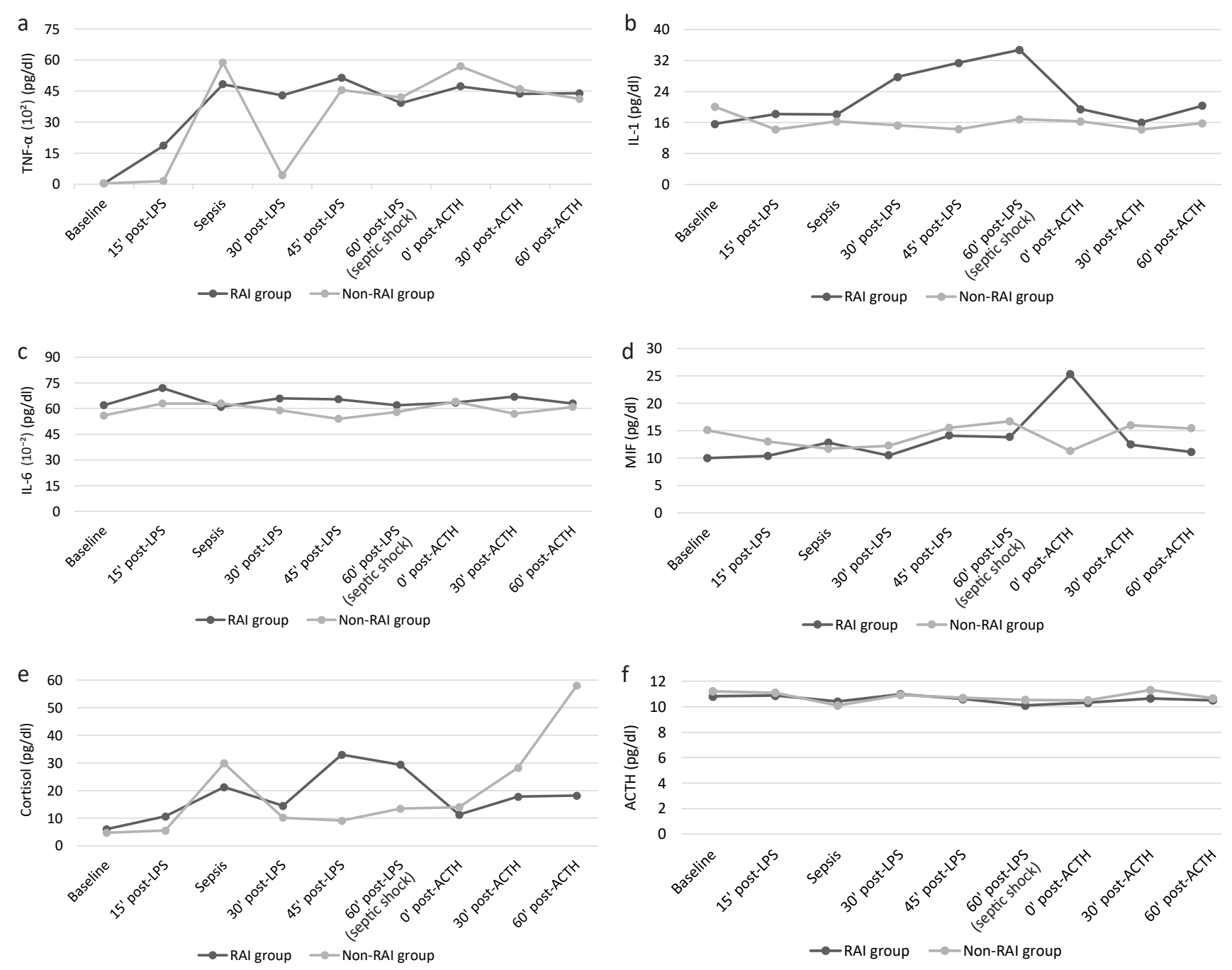

Figure 1. Dynamic changes of TNF- $\alpha$ (median), IL-1 (median), IL-6 (median), MIF (median), cortisol (mean), and ACTH (mean) in piglets with and without RAI. Baseline data were obtained before LPS injection. Sepsis appeared between 15 to 30 min after LPS injection. ACTH (Synacthen $\left.{ }^{\circledR}\right)$ injection was done immediately after septic shock. All piglets $(\mathrm{N}=19)$ had septic shock 60 min after LPS injection. RAI=relative adrenal insufficiency; IL=interleukin, TNF- $\alpha=$ tumor necrosis factor- $\alpha$, MIF=macrophage migration inhibitory factor; ACTH=adrenocorticotropin hormone Note: the scale of $\mathrm{x}$-axis is not proportional

The MIF level did not significantly suppress the ACTH level during septic shock $(p=0.648, r=0.112)$ and 60 min after the synacthen test $(p=0.096, r=0.393)$. The MIF level was correlated with ACTH during the sepsis condition $(p<0.001, r=0.736)$. MIF levels were inversely correlated with cortisol levels at $60 \mathrm{~min}$ after the synacthen stimulation test $(p=0.042, r=-0.471)$.

\section{Histopathological examination}

Histopathological examination showed inflammatory cell infiltration and hemorrhage in the adrenal gland in both the RAI and non-RAI groups, which indicated that the inflammatory process played a role in septic shock. Inflammatory cell infiltration was also observed in the hypothalamus and the pituitary glands in both groups. Immunohistochemical staining of the RAI group revealed that only the hypothalamus was positive for IL-1, IL-6, TNF- $\alpha$, and MIF, indicating that the ongoing inflammatory process resulted in a relatively temporary adrenal insufficiency; hence, the lack of IL-1, IL-6, TNF- $a$, and MIF staining after the monitoring period ended (Figure 2).

\section{DISCUSSION}

RAl is a condition in which, despite a maximally ACTH-activated adrenal cortex in response to critical illness, the cortisol production is still insufficient in response to general glucocorticoid receptor 

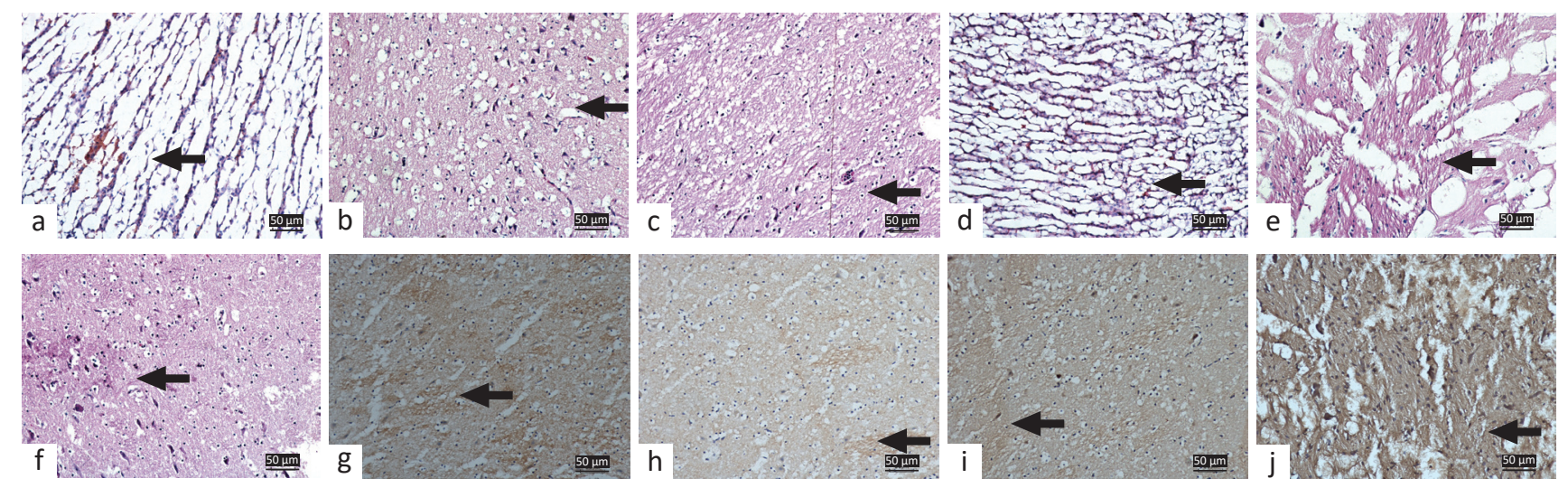

Figure 2. Hematoxylin and eosin staining of inflammatory cells infintration (black arrow) in: (a) adrenal gland, (b) pituitary gland, and (c) hypothalamus tissue in the RAI group; and (d) adrenal gland, (e) pituitary gland, and (f) hypothalamus in the non-RAI group. Immunohistochemistry staining using antibodies (black arrow) of: (g) IL-1; (h) IL-6; (i) TNF-a; and (j) MIF in hypothalamus tissue in the RAI group only. RAI=relative adrenal insufficiency; IL=interleukin, TNF- $\alpha=$ tumor necrosis factor- $\alpha$, MIF=macrophage migration inhibitory factor

activation to maintain hemodynamic stability. ${ }^{13}$ In this study, the incidence of RAI was $63 \%$ (12/19 piglets). The cortisol level was lower in the RAI group compared with the non-RAl group during septic shock. After the corticotropin stimulation test, the cortisol levels in the RAI group were also lower compared with the non-RAl group. These results showed that in the RAI group, the cortisol levels were insufficient to maintain hemodynamic stability. Our result was similar to a study by Singh et $\mathrm{a}^{14}$ in pediatric subjects, which reported the incidence of RAl as 40-65\%. Meanwhile, Menon et al ${ }^{15}$ found $30.2 \%$ RAl cases with baseline cortisol levels $28.6 \mu \mathrm{g} / \mathrm{dl}$. The difference of lower incidence of RAI, might be due to use of a lower dose of synacthen $(1 \mu \mathrm{g}$ synacthen). Rady et al ${ }^{16}$ also reported a RAl incidence of $33.3 \%$ using the same dose of synacthen in this study. Hebbar et $\mathrm{al}^{17}$ reported a $50 \%$ incidence of RAI. In Cipto Mangunkusumo Hospital, the incidence of $\mathrm{RAl}$ in pediatric patients was reported as $20 \% .^{18}$ These differences from our results may be due to different responses in piglets compared with humans and the time taken to perform the corticosteroid stimulation test. In this study septic shock was managed by administering fluid resuscitation, and inotropic drugs. Hydrocortisone was also given to overcome RAI.

Cortisol acts as a stimulant of the immune response at low concentrations and a suppressor of the immune response at high concentrations. ${ }^{19}$ The basal levels of cortisol can sensitize cells to harmful stimuli, even in the absence of inflammation, by increasing the expression of cytokine receptors, pattern recognition receptors, and complement factors. ${ }^{19}$ In this study, the cortisol levels in both groups were low from the $15^{\text {th }}$ to the $30^{\text {th }}$ min, which might be required to stimulate an immune response.

Systemic inflammation may cause a breakdown of the blood-brain barrier (BBB), facilitating the traffic of blood-borne cytokines to deep brain structures. In an animal study, the peripheral administration of endotoxin resulted in the expression of IL-1 and TNF- $\alpha^{20}$ In patients with septic shock, postmortem examination suggested overexpression of IL-1 and TNF- $\alpha$ in the hypothalamic nuclei. $^{20}$ In our study, the IL-1 levels during septic shock were similar in both groups after endotoxin administration as well as after the corticotropin stimulation test. In both groups, the IL-1 increase was followed a cortisol increase, although the cortisol levels in the RAI group were higher than in the non-RAI group. In the sepsis condition, the IL-1 level was correlated with the cortisol level $(p<0.001$, $r=0.728$ ), indicating that IL-1 played a role in increasing cortisol levels, which was attributed to activation of the HPA axis. IL-1 and TNF- $a$ act synergistically to induce a shock-like state characterized by vascular permeability, severe pulmonary edema, and hemorrhage. ${ }^{21}$ IL-1 was also identified as mediator for the development of fever. ${ }^{21}$ In our study, IL-1 levels increased in the $30^{\text {th }}$ min in the RAI and nonRAI groups, and IL-1 levels were correlated with the cortisol levels in the sepsis condition. Based on this result, IL-1 was involved in stimulating cortisol production, which, in turn, could stimulate immune and inflammation responses, which is needed in the 
sepsis condition. In our study, IL-1 levels decreased after the corticotropin stimulation test so that the inflammatory response was not excessive. IL-1 could also indirectly increase the cortisol level due to IL-6 production. Our immunohistochemical investigations showed IL-1 in the hypothalamic region, which proved that IL-1 played a role in activating the HPA axis to release corticotropin-releasing hormone $(\mathrm{CRH})$ and vasopressin from the hypothalamus.

IL-6 is also an activator of the HPA axis. This inflammatory mediator can reach the portal circulation in the median eminence, located outside the BBB, via the anterior hypophyseal arteries. ${ }^{13,20}$ In our study, IL- 6 was correlated with ACTH at the $60^{\text {th }}$ min, which indicated that IL-6 played a role in stimulating the HPA axis. IL- 6 was also correlated with cortisol at the $60^{\text {th }} \mathrm{min}$, which indicated that IL-6 also stimulated cortisol production by increasing ACTH production. Furthermore, the IL-6 level in the RAI group was higher than in the non-RAl group from the $15-60^{\text {th }}$ min, which showed that IL-6 acted as a stimulator of inflammation from the beginning. However, a significant difference was observed between the RAl group and the non-RAl group at the $45^{\text {th }}$ min which was related to the immune response to eliminate the endotoxin. The increased IL-6 level also showed that it played a role in inducing cortisol production so that an excessive response to inflammation did not occur. Matsumoto et al ${ }^{22}$ found that IL- 6 on day-2 and -4 in the critically ill patients was significantly increased compared to those in the non-critically ill patients. IL-6 serves as an important mediator during the acute phase of response to inflammation in sepsis. Song et $\mathrm{al}^{23}$ found that IL-6 could discriminate sepsis from control with cut-off value $52.60 \mathrm{pg} / \mathrm{ml}, 80.4 \%$ sensitivity, and $88.9 \%$ specificity. Karrow et $\mathrm{al}^{24}$ showed that the LPS level could induce a peak cortisol level in 2 hours and a peak IL-6 level in 1 hour. Our immunohistochemical investigations found IL- 6 in the hypothalamus, which proved that IL- 6 might play a role in hypothalamic stimulation.

The proinflammatory cytokine TNF- $a$ also contributes to activating the HPA axis. TNF- $\alpha$ overexpression in hypothalamic nuclei has been reported in postmortem examinations in patients with septic shock. ${ }^{13,20}$ In our study, immunohistochemical staining showed that TNF- $\alpha$ was found in the hypothalamic region, proving that TNF-a was involved in stimulating the hypothalamic region. Animal experiments have suggested that TNF- $\alpha$ induced release of corticosterone is a $\mathrm{CRH}$-dependent mechanism. ${ }^{20}$ TNF- $\alpha$ is also produced in adrenal tissues by resident macrophages and adrenocortical cells. ${ }^{20}$ The presence within the adrenals of TNF- $a$ and its receptors in the adrenal glands suggests that this cytokine plays a role in adrenal function in stimulatory steroidogenesis. ${ }^{20}$ In our study, there was no correlation between TNF- $\alpha$ and both cortisol and $\mathrm{ACTH}$, indicating that cortisol production occurred indirectly by stimulating the hypothalamic region. Cortisol production in the early stages of inflammation can be increased due to an increase of IL-1. ${ }^{23}$ Our study found that TNF- $a$ was higher from the $15-30^{\text {th }} \mathrm{min}$ during septic shock in the RAI group than in the nonRAl group. The release of TNF- $\alpha$ from macrophages begins within 30 min after the inciting event, following gene transcription and RNA translation. ${ }^{21}$ In our study, the TNF-a levels in the non-RAI group were increased after the $15^{\text {th }}$ min during septic shock and decreased after septic shock.

MIF was initially described as a $\mathrm{T}$ lymphocyte product that inhibited the random migration of macrophages. ${ }^{7}$ MIF functions as corticosteroid contraregulator and modulates immune responses and inflammation. ${ }^{7,25}$ A low level of corticosteroids can induce MIF release, which reduces immune suppression and the anti-inflammatory effects of glucocorticoids..$^{6,7}$ In our study, MIF levels were found to correlate with ACTH levels in the sepsis condition, leading to the production of ACTH in the pituitary gland, which stimulated cortisol production. ${ }^{6,7}$ We also found that MIF levels were correlated with cortisol levels at the $60^{\text {th }}$ min after corticotropin injection, indicating that MIF functioned as a corticosteroid contraregulator, maintaining them at lower concentrations so that cortisol could exert its anti-inflammatory effects. We observed higher MIF levels in the RAI group, indicating macrophage accumulation at the inflammation site. In our study, the MIF levels in the RAI group were higher than in the non-RAl group in septic shock condition. An exaggerated early-phase response, which occurs due to the expression of proinflammatory mediators, can lead to early mortality as a result of shock. ${ }^{25}$ This can be indicated by increased levels of MIF, which acts as a key mediator of the systemic inflammatory response and is a predictive factor of early death from severe sepsis. ${ }^{25}$ 
The role of IL-1, IL-6, TNF- $\alpha$, and MIF in inflammation and cortisol production in the RAl group can be seen in Figure 1. In this group, IL-1 increased to stimulate IL-6, in the beginning to the $15^{\text {th }} \mathrm{min}$. TNF-a levels also increased in the $15^{\text {th }} \mathrm{min}$ as proinflammatory mediator. The higher concentration of TNF- $\alpha$ in the RAI group compared with the non-RAl suppressed cortisol production. After 15 min, IL- 6 concentration levels decreased in RAI group compared with the nonRAI group. The IL-1 levels continued to increase, which stimulated cortisol production. The cortisol levels from the $30-60^{\text {th }}$ min were higher compared with the ACTH levels. A low plasma ACTH in the presence of high plasma cortisol concentrations has been interpreted as non-ACTH driven cortisol production, in which cytokines could play a role. ${ }^{13}$ The increased availability of systemic cortisol during critical illness seems to be largely driven by decreased cortisol breakdown in the liver and kidneys. ${ }^{19}$ In the septic shock conditions in our study, MIF levels were increased so that plenty of inflammatory cells would become concentrated at the inflammatory site. After the corticotropin stimulation test, cortisol levels were not increased, showing that the adrenal gland was no longer capable of cortisol production. The levels of TNF- $\alpha$ also contributed to the suppression of cortisol production and prolonging the septic shock condition. The ACTH levels were relatively stable from the onset of sepsis until the septic shock condition in the RAI group. In this case, the proinflammatory cytokines might have played a lesser role in hypothalamic stimulation or other cytokines could have been involved in suppressing ACTH levels. ${ }^{20}$

The role of IL-1, IL-6, TNF- $\alpha$, and MIF in inflammation, cortisol, and ACTH production in the RAI and non-RAl group is shown in Figure 1. In this group, IL-1 levels also increased to stimulate IL-6, which subsequently stimulated TNF-a production. The IL- 6 increase also stimulated cortisol production to prevent an overt inflammation reaction. In the $15^{\text {th }} \mathrm{min}$, IL-1 decreased in the non-RAI group, which showed that the stimulatory effect of IL-1 was less prominent in the $15^{\text {th }}$ min. Cortisol levels increased after septic shock, indicating its anti-inflammatory role. In the $30^{\text {th }} \mathrm{min}$, IL-6 levels decreased to prevent an overt inflammation reaction. The IL- 6 levels increased again after the $30^{\text {th }} \mathrm{min}$, and TNF-a levels decreased after septic shock so that cortisol could assert its antiinflammatory role. MIF levels also increased after the $60^{\text {th }}$ min, resulting in an accumulation of inflammatory cells. In our study, IL-6 mainly increased in the RAI group.

RAI occurs when the degree of HPA axis activation is assumed to be insufficient to cover cortisol requirements for survival, even when plasma cortisol levels are higher than under healthy conditions. Hypotension refractory to fluid resuscitation and vasopressors may be signs of RAl.3,6,13 Diagnostic criteria for RAI is based on an increased cortisol increment response of $<9 \mu \mathrm{g} / \mathrm{dl}$ after the injection of $250 \mu \mathrm{g} \mathrm{ACTH.9,13} \mathrm{The} \mathrm{corticotropin} \mathrm{stimulation}$ test is sometimes difficult to perform due to the extremely rare availability of synthetic ACTH. Thus, other parameters are required to diagnose RAI. The differences in cytokines between RAI and non-RAI that we observed in our study should be considered as potential biomarkers of RAI or as indicators of the risk of RAI.

Our study has several limitations. Before undertaking this study, we conducted a preliminary study to assess normal vital signs in piglets during sepsis until septic shock. We also assessed $\mathrm{Cl}$, intrathoracic blood volume index, and extravascular lung water index to determine the septic shock condition. This was the first time that our procedure was conducted in the animal laboratory used in our study. The other problems encountered included difficulties in inserting the PiCCO catheter and central venous catheter because they were was not designed for piglets, sedation problems with the piglets, and hypoglycemia due to fasting before the procedure. Our study was conducted in piglets, which might not be similar to humans. This study was also an experimental study, and a different study design is necessary to conduct similar research in humans. Further research needs to be conducted to determine the MIF cut-off point that can cause RAI.

In conclusion, the levels of IL-1, IL-6, and TNF- $\alpha$ were similar during septic shock. The TNF-a level in our piglet model of septic shock with RAI was increased at the $15^{\text {th }}$ and $30^{\text {th }} \mathrm{min}$. The MIF level in the piglet model of septic shock with RAI was significantly increased during septic shock. The IL-6 level in the piglet model of septic shock with RAl was higher at the $45^{\text {th }}$ min. Immunohistochemistry revealed that IL-1, IL-6, TNF-a, and MIF were only positive in the hypothalamus from the piglet model of septic shock with RAI. Hydrocortisone treatment can be given earlier, based

mji.ui.ac.id 
on IL-6, TNF- $\alpha$, and MIF levels in pediatric patients with septic shock. Cytokines IL-6, TNF-a, and MIF could be used as predictors of the incidence of RAI in septic shock.

\section{Conflict of Interest}

Jose R. L. Batubara is the business manager but was not involved in the review or decision process for the article.

\section{Acknowledgment}

We acknowledge the support and permission by Prof. Srihadi Agungpriyono, BVM, Dean of Faculty of Veterinary Medicine, Institut Pertanian Bogor, Bogor, Sri Estuningsih, PhD, Msi, APVet, Head of Animal Research Ethics Committee with all staff, and also Heri Wibowo BSc M.Biomed PhD, Neneng Gusniarti BSc, Abdiyanah Mahyuni, Sri Supriyanto from Integrated Laboratory, Faculty of Medicine Universitas Indonesia. We also acknowledge the support during research procedure by Riki Suwandi BVM, MSi, Dwi Utari Handayani, BVM, Bangkit BVM MSi, Heryudianto BVM from Faculty of Veterinary Medicine, also Silvia Prabandari BVM APVet, Satyawati BVM, Primate Study Centre, Institut Pertanian Bogor for the support of histopathology procedure.

\section{Funding Sources}

None.

\section{REFERENCES}

1. Latief A, Chairulfatah A, Alam A, Pudjiadi AH, Somasetia DH, Djatnika Setiabudi D. Diagnosis and management of sepsis in children. In: Latief A, editor. National Guidelines for Medical Services IDAI. 1st ed. Jakarta: IDAI; 2016. p. 1-47. Indonesian.

2. Vila-Pérez D, Jordan- García L. Relative adrenal insufficiency in pediatric septic shock. J Pediatr Intensive Care. 2015;4(3):129-37.

3. Linck Júnior A, Rego Filho Ede A, Moriya LK, Cardoso JR. Adrenal insufficiency in children with sepsis. Rev Bras Intensiva. 2011;23(4):478-83.

4. Widodo AD, Tumbelaka AR. Use of steroids in the management of sepsis in an evidance-based case analysis. Sari Pediatri. 2010;11(6):387-94. Indonesian.

5. Silverman MN, Sternberg EM. Glucocorticoid regulation on inflammation and its functional correlates: from HPA axis to glucocorticoid receptor dysfunction. Ann N Y Acad Sci. 2012;1261:55-63.

6. Van den Berghe G. Novel insights in the HPA-axis during critical illness. Acta Clin Belg. 2014;69(6):397-406.

7. Grieb G, Merk M, Bernhagen J, Bucala R. Macrophage migration inhibitory factor (MIF): a promising biomarker. Drug News Perspect. 2010;23(4):257-64.

8. Stevens JP, Haase E, Churchill T, Bigam DL, Cheung PY. Resuscitation with $21 \%$ or $100 \%$ oxygen is equally effective in restoring perfusion and oxygen metabolism in the liver of hypoxic newborn piglets. Shock. 2007;27(6):657-62.

9. Chapados I, Chik CL, Cheung PY. Plasma cortisol response to ACTH challenge in hypoxic newborn piglets resuscitated with
$21 \%$ and $100 \%$ oxygen. Shock. 2010;33(5):519-25.

10. Dewi R, Supriyatno B, Madjid AS, Gunanti, Lubis M. The effects of colloids or crystalloids on acute respiratory distress syndrome in swine (Sus scrofa) models with severe sepsis: analysis on extravascular lung water, IL-8, and VCAM-1. Med J Indones. 2016;25(1):33-8.

11. Dellinger RP, Levy MM, Rhodes A, Annane D, Gerlach H, Opal $S M$, et al. Surviving sepsis campaign: international guidelines for management of severe sepsis and septic shock. Crit Care Med. 2013;41(2):580-637.

12. Stenman UH. Standardization of hormone determinations. Best Pract Res Clin Endocrinol Metab. 2013;27(6):823-30.

13. Boonen E, Van den Berghe $G$. Endocrine responses to critical illness: novel insights and therapeutic implications. J Clin Endocrinol Metab. 2014;99(5):1569-82.

14. Singh SN, Rathia SK, Awasthi S, Singh A, Bhatia V. Salivary cortisol estimation to assess adrenal status in children with fluid unresponsive septic shock. Indian Pediatr. 2013;50(7):681-4.

15. Menon K, Ward RE, Lawson ML, Gaboury I, Hutchison JS, Hébert PC; Canadian Critical Care Trials Group. A prospective multicentre study of adrenal function in critically ill children. Am J Respir Crit Care Med. 2010;182(2):246-51.

16. Rady HI, Aly YS, Hafez M, Bazaraa HM. Adrenocortical status in infants and childrenwith sepsis and septic shock. Gaz Egypt Paediatr Assoc. 2014;62(1):18-23.

17. Hebbar kB, Stockwell JA, Leong T, Fortenberry JD. Incidence of adrenal insufficiency and impact of corticosteroid supplementation in critically ill children with systemic inflammatory syndrome and vasopressor-dependent shock. Crit Care Med. 2011;39(5);1145-50.

18. Rachmawati RI, Widodo DP, Tridjaja B, Pudjiadi AH. Adrenal function in sepsis in a pediatric intensive care unit. 2011;12(6):426-32. Indonesian.

19. Téblick A, Peeters B, Langouche L, Van den Berghe G. Adrenal function and dysfunction in critically ill patients. Nat Rev Endocrinol. 2019;15:417-27.

20. Annane D. The role of ACTH and corticosteroids for sepsis and septic shock: an update. Front Endocrinol (Lausanne). 2016;7:70.

21. Schulte W, Berrnhagen J, Bucala R. Cytokines in sepsis: potent immunoregulators and potential therapeutic targets-an updated view. Mediators Inflamm. 2013;2013;165974.

22. Matsumoto H, Ogura H, Shimizu K, Ikeda M, Hirose T, Matsuura $\mathrm{H}$ et al. The clinical importance of a cytokine network in the acute phase of sepsis. Sci Rep. 2018;8:13995.

23. Song J, Park DW, Moon S, Cho HJ, Park JH, Seok H, et al. Diagnostic and prognostic value of interleukin-6, pentraxin 3 and procalcitonin levels among sepsis and septic shock patients: a prospective controlled study according to the sepsis-3 definitions. BMC Infect Dis. 2019;19(1):968.

24. Karrow NA, You Q, McNicoll C, Hay J. Activation of the ovine hypothalamic-pituitary-adrenal axis and febrile response by interleukin-6: a comparative study with bacterial lipopolysaccharide endotoxin. Can J Vet Res. 2010;74(1):30-3.

25. Chuang Ty, Chang HT, Chung KP, Cheng HS, Liu CY, Liu YCet al. High levels of serum macrophage migration inhibitory factor and interleukin 10 are associated with a rapidly fatal outcome in patients with severe sepsis. Int J Infect Dis. 2014;20:13-7. 